\title{
Statistically-driven Metabolite and Lipid Profiling of Patients from the Undiagnosed Diseases Network
}

\author{
Bobbie-Jo M. Webb-Robertson ${ }^{\dagger *}$, Kelly G. Stratton ${ }^{\ddagger}$, Jennifer E. Kyle ${ }^{\dagger}$, Young-Mo Kim ${ }^{\dagger}$ Lisa M. \\ Bramer $^{\ddagger}$, Katrina M. Waters ${ }^{\dagger}$, David M. Koeller ${ }^{\ddagger}$, Thomas O. Metz ${ }^{\dagger *}$ \\ †Biological Sciences Division, Pacific Northwest National Laboratory, Richland WA 99352, USA \\ ‡Computing Analytics Division, Pacific Northwest National Laboratory, Richland WA 99352, USA \\ \$Molecular and Medical Genetics, School of Medicine, Oregon Health and Science University, Portland, OR 97239, USA
}

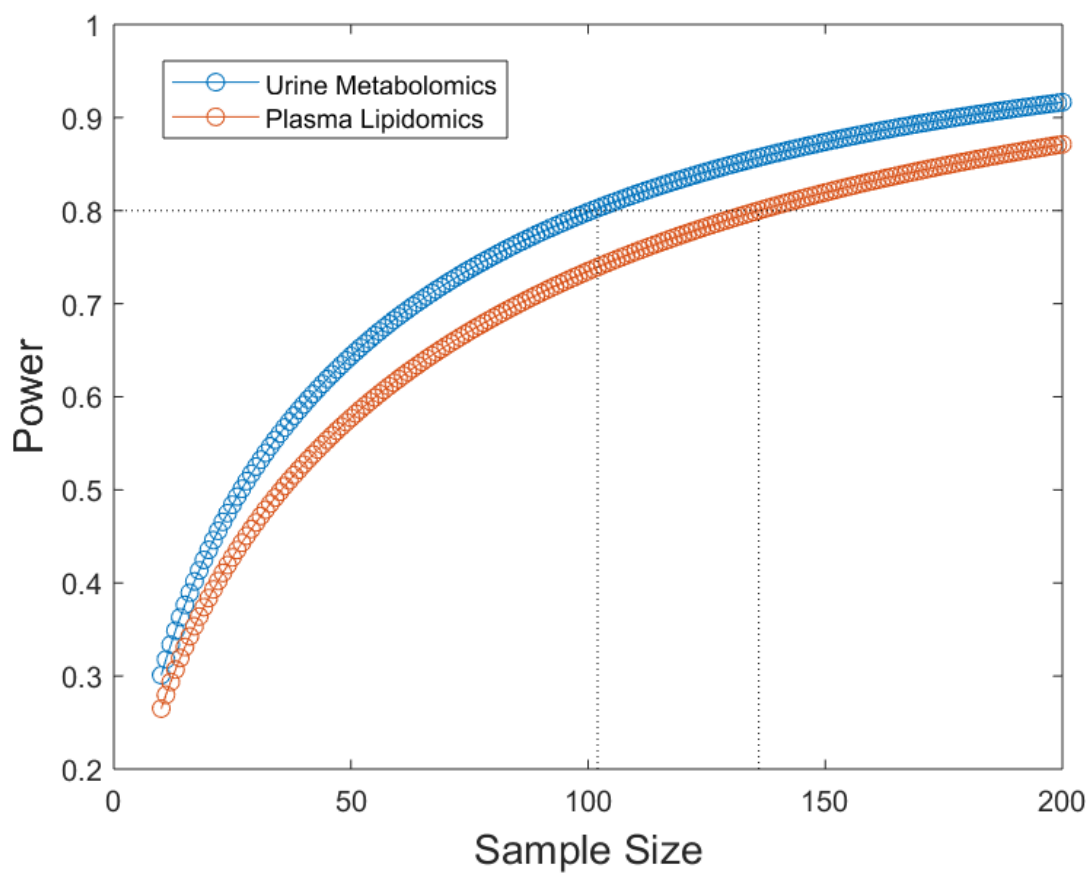

Supplemental Figure 1: The power curve of the sample size versus the power based on testing for a significant difference in abundance based on a t-test, type 1 error of 0.05 and a two-fold change in abundance on the log scale. The urine data consisted of a study with 188 subjects and 180 metabolites, including knowns and unknowns. The plasma data consisted of a study with 100 subjects and 149 lipids. A standard deviation was computed for each metabolite and lipid and the standard deviation was selected that included at least 95\% of the features, i.e., the 95-th quantile of the distribution of the 180 and 149 standard deviations, respectively for the metabolites and lipids. This standard deviation was used as the basis for the power calculation generating the power curves assuming a UDN sample size of 2. 